\title{
EQUIVARIANT MORSE THEORY FOR FLOWS AND AN APPLICATION TO THE $N$-BODY PROBLEM
}

\author{
FILOMENA PACELLA
}

\begin{abstract}
In this paper, using Conley's index and equivariant cohomology, some Morse type inequalities are deduced for a flow equivariant with respect to the action of a compact topological group.

In the case of a gradient flow induced by a nondegenerate smooth function these inequalities coincide with those described by $\mathrm{R}$. Bott.

The theory is applied to the study of the central configurations of $N$-bodies.
\end{abstract}

Introduction. When a flow on a topological space is subject to some symmetry there are at least three ways of obtaining Morse inequalities [16]. With simple examples it is possible to prove that, in the case of a nonfree action of a symmetry group, these three approaches give different information [16].

In this paper we describe the equivariant Morse theory for flows in the presence of a symmetry due to the action of a compact topological group.

The theory presented here generalizes the one described in [1 and 4] for a gradient flow induced by a nondegenerate smooth function. In particular, our extension allows us to treat the case of functions having degenerate critical orbits.

We point out that a different approach to the study of equivariant gradient flows, in the presence of particular symmetries, is described in $[2,9,10]$ using the cohomological or geometrical index as a replacement for the Ljusternik-Schnirelmann category.

In $\$ 3$ we apply the equivariant Morse theory to the $N$-body problem, showing that the Morse relations obtained in [15] hold also when the potential energy has some degenerate critical orbits.

Moreover we prove that the Morse inequalities for the spatial problem imply the existence of many more central configurations than one could deduce from the Morse inequalities for the planar problem [14]. This is relevant for the question of the existence of spatial central configurations.

We end $\$ 3$ by proving that the collinear problem is a repeller in the gradient flow induced by the potential energy.

ACKNOwLEDGment. This paper is dedicated to Charles Conley who suggested the topic discussed here, in [15], and in [16].

Received by the editors March 11, 1985.

1980 Mathematics Subject Classification. Primary 57S10, 58F25, 70F10.

Key words and phrases. Conley's index, group actions, Morse inequalities, $N$-body problem.

Work supported by M. P. I. (40\%, 1985). 
I am indebted to him for the insights, advice and encouragement he offered during my stay at the Mathematics Research Center of the University of WisconsinMadison and at the Institute for Mathematics and its Applications of the University of Minnesota-Minneapolis.

1. Preliminaries. Let $\Gamma$ be a Hausdorff topological space and the map $(\gamma, t) \rightarrow \gamma \cdot t$ from $\Gamma \times \mathbf{R}$ to $\Gamma$ a flow on $\Gamma[7,8,16,17]$.

We recall [8] that a locally compact Hausdorff subspace $X$ of $\Gamma$ is a local flow if for every $\gamma \in X$ there is a neighborhood $U \subset \Gamma$ of $\gamma$ and an $\varepsilon>0$ such that $(X \cap U) \cdot[0, \varepsilon) \subset X$.

We suppose that there is a (left) action of a compact topological group $G$ on $\Gamma$ [6, 16].

A subset $Y \subseteq \Gamma$ is called $G$-invariant if

$$
g \in G \text { and } \gamma \in Y \Rightarrow g \gamma \in Y
$$

where $g \gamma$ denotes the element of $Y$ obtained from $\gamma$ using the action of $g \in G$.

We say that the flow on $\Gamma$ is equivariant if

$$
(g \gamma) \cdot t=g(\gamma \cdot t), \quad \gamma \in \Gamma, g \in G, t \in \mathbf{R} .
$$

This allows us to define a flow on the quotient $\Gamma / G$ in the following way:

$$
[\gamma] \cdot t=[\gamma \cdot t], \quad[\gamma] \in \Gamma / G, t \in \mathbf{R},
$$

where $[\gamma]$ is the orbit of a point $\gamma \in \Gamma$ under the action of $G$.

The flow (1.2) is well defined because if $\gamma^{\prime}$ belongs to the orbit of $\gamma$, then $\gamma^{\prime}=g \gamma$, for some $g \in G$, and consequently

$$
\left[\gamma^{\prime}\right] \cdot t=\left[\gamma^{\prime} \cdot t\right]=[(g \gamma) \cdot t]=[g(\gamma \cdot t)]=[\gamma \cdot t]=[\gamma] \cdot t .
$$

It is easy to prove the following proposition.

Proposition 1.1. Let $X$ be a $G$-invariant local flow in the G-equivariant flow on $\Gamma$ and I a G-invariant isolated invariant set in $X$. Then

(i) $X / G$ is a local flow in $\Gamma / G$,

(ii) $I / G$ is an isolated invariant set in $X / G$,

(iii) if $\left(N, N^{-}\right)$is an index pair for I with $N$ and $N^{-} G$-invariant then $\left(N / G, N^{-} / G\right)$ is an index pair for $I / G$.

Proof. (i) $X / G$ is locally compact and is an Hausdorff subspace of $\Gamma / G$ because $G$ is a compact topological group [6].

Take $[\gamma] \in X / G, \gamma \in X$. Since $X$ is a local flow in $\Gamma$, there exists a neighborhood $U_{\gamma} \subset \Gamma$ and $\varepsilon>0$ such that $\left(X \cap U_{\gamma}\right) \cdot[0, \varepsilon) \subset X$.

Since $G$ is compact any neighborhood of a $G$-invariant set contains a $G$-invariant neighborhood [6, Ex. 9, p. 69], so we can suppose that $U_{\gamma}$ is $G$-invariant. Then $U_{\gamma} / G$ is a neighborhood of $[\gamma]$ in $\Gamma / G$ and $\left(X / G \cap U_{\gamma} / G\right) \cdot[0, \varepsilon) \subset X / G$.

(ii) To say that $I$ is an isolated invariant set in $X$ means $[7,8]$ that there exists a compact $N \subset X$ such that $I \subset$ int $N$ (relative to $X$ ) and $I=I(N)$ where $I(N)$ is the maximal invariant set contained in $N$, that is $I(N)=\{\gamma \in N \mid \gamma \cdot \mathbf{R} \subset N\}$.

As above we can suppose that $N$ is $G$-invariant. Then $I / G \subset \operatorname{int}(N / G), N / G$ is compact and $I / G=\{[\gamma] \in N / G \mid[\gamma] \cdot \mathbf{R} \subset N / G\}$. 
(iii) We recall that a compact pair $\left(N, N^{-}\right)$is an index pair for the isolated invariant set $I[8]$ if

(a) $\operatorname{cl}\left(N \backslash N^{-}\right)$is an isolating neighborhood of $I$,

(b) $\gamma \in N^{-}$and $\gamma \cdot[0, t] \subset N$ imply $\gamma \cdot[0, t] \subset N^{-}$,

(c) if $\gamma \in N$ and $\gamma \cdot \mathbf{R}^{+} \not \subset N$ then there exists $\bar{t} \geqslant 0$ such that $\gamma \cdot[0, \bar{t}] \subset N$ and $\gamma \cdot \bar{t} \in N^{-}$.

Since $N$ and $N^{-}$are $G$-invariant and compact, $N \backslash N^{-}$is $G$-invariant and relatively compact. Thus, by (ii) $\operatorname{cl}\left(N / G \backslash N^{-} / G\right)=\operatorname{cl}\left(\left(N \backslash N^{-}\right) / G\right)=$ $\left(\operatorname{cl}\left(N \backslash N^{-}\right)\right) / G$ is an isolating neighborhood of $I / G$.

Moreover, if $\alpha \in N^{-} / G$, then $\alpha=[\gamma]$ for some $\gamma \in N^{-}$.

From $\alpha \cdot[0, \bar{t}] \subset N / G$ and (b) we have $\forall t \in[0, \bar{t}]$

$$
\begin{aligned}
{[\gamma] \cdot t } & =[\gamma \cdot t] \in N / G \Rightarrow \gamma \cdot t \in N \\
& \Rightarrow \gamma \cdot t \in N^{-} \Rightarrow[\gamma \cdot t]=[\gamma] \cdot t \in N^{-} / G .
\end{aligned}
$$

Finally, if $\alpha \in N / G$ and $\alpha \cdot \mathbf{R}^{+} \not \subset N / G$, then $\alpha=[\gamma]$, for some $\gamma \in N$ and there exists $t \in R^{+}$such that $[\gamma] \cdot t=[\gamma \cdot t] \notin N / G$. This implies that $\gamma \cdot \mathbf{R}^{+} \not \subset N$. Thus, from (c) there exists $\bar{t} \geqslant 0$ such that $\forall t \in[0, \bar{t}], \gamma \cdot t \in N$ and $\gamma \cdot \bar{t} \in N^{-}$. Consequently $\alpha \cdot t=[\gamma \cdot t] \in N / G, \forall t \in[0, \bar{t}]$, and $\alpha \cdot \bar{t}=[\gamma \cdot \bar{t}] \in N^{-} / G$.

REMARK 1.1. Given the $G$-invariant isolated invariant set $I$ it is always possible to construct an index pair $\left(N, N^{-}\right)$with $N$ and $N^{-} G$-invariant. In fact, since $I / G$ is an isolated invariant set in $X / G$, there exists an index pair $\left(M, M^{-}\right)$of $I / G$ in $X / G$. Then, denoted by $\pi$ the canonical projection of $\Gamma$ on $\Gamma / G,\left(\pi^{-1}(M), \pi^{-1}\left(M^{-}\right)\right)$ is an index pair for $I$ in $X$.

From now on we will denote by $S$ a $G$-invariant isolated invariant set in the $G$-invariant local flow $X$.

Proposition 1.2. If $\left(M_{1}, \ldots, M_{n}\right)$ is an admissible ordering of a Morse decomposition of $S$, given by $G$-invariant Morse sets, then $\left(M_{1} / G, \ldots, M_{n} / G\right)$ is an admissible ordering of a Morse decomposition of $S / G$.

Proof. A Morse decomposition of $S[7,8]$ is a finite collection $\left\{M_{\pi}\right\}_{\pi \in P}$ of disjoint, compact, invariant subsets $M_{\pi} \subset S$ which can be ordered $\left(M_{1}, \ldots, M_{n}\right)$ in such a way that for every $\gamma \in S \backslash \bigcup_{1 \leqslant j \leqslant n} M_{j}$ there are indices $i<j$ such that $\omega(\gamma) \subset M_{i}$ and $\omega^{*}(\gamma) \subset M_{j}$.

The $\omega$-limit sets, $\omega(\gamma)$ and $\omega^{*}(\gamma)$ are defined to be, respectively, the maximal invariant set in the closure of $\gamma \cdot[0,+\infty)$ and $\gamma \cdot(-\infty, 0]$.

Since each $M_{j}$ is $G$-invariant and $G$ is compact it is easy to see that $\left\{M_{\pi} / G\right\}_{\pi \in P}$ is a finite collection of disjoint, compact, invariant subsets of $S / G$.

Now let us take for $\left\{M_{\pi} / G\right\}_{\pi \in P}$ the same ordering as for $\left\{M_{\pi}\right\}_{\pi \in P}$ and prove that this ordering is admissible.

To do this it is enough to show that if $\gamma$ and $\gamma^{\prime}$ are in the same equivalence class, that is, $\gamma^{\prime}=g \gamma$, for some $g \in G$, then

$$
\omega\left(\gamma^{\prime}\right)=\omega(g \gamma)=g \omega(\gamma) \text { and } \omega^{*}\left(\gamma^{\prime}\right)=g \omega^{*}(\gamma) .^{1}
$$

\footnotetext{
${ }^{1}$ Given $A \subset \Gamma$, we denote by $g A, g \in G$, the set $g A=\{g x, x \in A\}$.
} 
In fact, if $\alpha \in S / G \backslash \cup_{1 \leqslant i \leqslant n} M_{i} / G$,

$$
\omega(\alpha)=\left(\bigcup_{g \in G} \omega(g \gamma)\right) / G,
$$

with $\gamma$ such that $[\gamma]=\alpha$, and so (1.3) together with the $G$-invariance of each $M_{\pi}$ implies the assertion.

Let us prove (1.3). By definition $\omega(g \gamma)$ is the maximal invariant set contained in $\operatorname{cl}(\{g \gamma \cdot[0, \infty)\})$.

Then we have

$$
\begin{aligned}
\omega(g \gamma) & =I(\operatorname{cl}(\{g \gamma \cdot[0, \infty)\}))=I(\operatorname{cl}(\{g(\gamma \cdot[0, \infty))\})) \\
& =I(g \operatorname{cl}(\{\gamma \cdot[0, \infty)\}))=g I(\operatorname{cl}(\{\gamma \cdot[0, \infty)\}))=g \omega(\gamma) .
\end{aligned}
$$

In the same way it is possible to prove that $\omega^{*}(g \gamma)=g \omega^{*}(\gamma)$.

REMARK 1.2. If $S$ is $G$-invariant and $\left(M_{1}, \ldots, M_{n}\right)$ is a Morse decomposition of $S$, it is possible to obtain a new Morse decomposition of $S$, built up by $G$-invariant Morse sets, putting together the sets $M_{i}$ which are in the same orbit.

Actually, if the group is connected, each isolated invariant set must be $G$ invariant.

2. Equivariant Morse theory. As in $\S 1$ we suppose that an equivariant flow is defined on the Hausdorff topological space $\Gamma$.

If $G$ is a compact topological group there exists a universal $G$-bundle characterized by having its total space $E$ contractible:

$$
\begin{gathered}
G \\
\downarrow \\
E \\
\downarrow \\
E / G=B G
\end{gathered}
$$

The space $B G$ is the so-called classifying space of $G$. The action of $G$ on $E$ is free $^{2}$ and $E$ is unique, up to homotopy.

Since the action of $G$ on $E$ is free, the diagonal action of $G$ on the product $\Gamma \times E$ defined by

$$
g(\gamma, e)=(g \gamma, g e), \quad g \in G, \gamma \in \Gamma, e \in E,
$$

is also free.

The flow can be extended to the product $\Gamma \times E$ in the trivial way:

$$
(\gamma, e) \cdot t=(\gamma \cdot t, e), \quad t \in \mathbf{R} .
$$

Of course this flow is $G$-equivariant on the product $\Gamma \times E$. Then it can be projected to a flow on the quotient space $(\Gamma \times E) / G=\Gamma_{G}$.

It is obvious that if $I$ is a $G$-invariant, invariant set for the flow on $\Gamma$, then $(I \times E) / G=I_{G}$ is an invariant set for the quotient flow in $\Gamma_{G}$.

The aim of this section is to obtain a kind of "Morse inequality" for this quotient flow.

\footnotetext{
${ }^{2}$ An action of $G$ on a space $T$ is said to be free if $g \in G$ and $g \neq 1 \Rightarrow g x \neq x \forall x \in T$.
} 
To do this, following the procedure of [8], we need a filtration of compact pairs. But, usually, in the universal bundle (2.1), E and $B G$ are realized as infinite dimensional manifolds, hence we cannot hope to construct compact pairs for any isolated invariant set.

Actually the definition itself of isolated invariant set requires the presence of a compact isolating neighborhood, so that we cannot even say that a set is an isolated invariant set in $\Gamma_{G}$.

Using a limit procedure we can still derive some "Morse inequalities".

When $G$ is a compact topological group, $E$ and $B G$ can be obtained as the limit of finite dimensional compact spaces

related to the bundles:

$$
E=\lim _{k \rightarrow \infty} E_{k}, \quad B G=\lim _{k \rightarrow \infty} B_{k} G,
$$

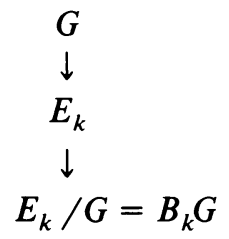

The action of $G$ on $E_{k}$ is free and hence the diagonal action on the product $\Gamma \times E_{k}$ is also free.

As before we extend the flow to the product $\Gamma \times E_{k}$ in the trivial way and we project it to the quotient $\left(\Gamma \times E_{k}\right) / G$.

Then we have

PROPOSITION 2.1. If I is a G-invariant isolated invariant set for the flow on $\Gamma$, then

(a) $\left(I \times E_{k}\right) / G$ is an isolated invariant set for the flow on $\left(\Gamma \times E_{k}\right) / G$.

(b) If $\left(N, N^{-}\right)$is an index pair for $I$ and $N$ and $N^{-}$are G-invariant, then $\left(\left(N \times E_{k}\right) / G,\left(N^{-} \times E_{k}\right) / G\right)=\left(N_{k}, N_{k}^{-}\right)$is an index pair for $\left(I \times E_{k}\right) / G$.

(c) If $\left(M_{1}, \ldots, M_{n}\right)$ is an admissible ordering of a Morse decomposition of I, given by G-invariant Morse sets, then $\left(\left(M_{1} \times E_{k}\right) / G, \ldots,\left(M_{n} \times E_{k}\right) / G\right)$ is an admissible ordering of a Morse decomposition of $\left(I \times E_{k}\right) / G$.

Proof. (a) and (b) follow from (ii) and (iii) of Proposition 1.1 because it is easy to see that $I \times E_{k}$ is an isolated invariant set in $\Gamma \times E_{k}$ and $\left(N \times E_{k}, N^{-} \times E_{k}\right)$ is a $G$-invariant index pair for $I \times E_{k}$.

Part (c) follows from Proposition 1.2 because $\left(M_{1} \times E_{k}, \ldots, M_{n} \times E_{k}\right)$ is an admissible ordering of a Morse decomposition of $\left(I \times E_{k}\right)$.

If $E_{k}$ is a Hausdorff space the previous proposition can be formulated for the local flow $\left(X \times E_{k}\right) / G$.

Let us denote by $S$ a $G$-invariant isolated invariant set in $\Gamma$ and by $\left(M_{1}, \ldots, M_{n}\right)$ a Morse decomposition of $S$ built up by $G$-invariant sets.

By virtue of Proposition 2.1, for each $k \in N$ we can write the following Morse inequalities [8]:

$$
\sum_{j=1}^{n} P_{t}\left(h_{k}\left(M_{j}\right)\right)=P_{t}\left(h_{k}(S)\right)+(1+t) Q_{t}^{k}
$$


where $h_{k}(S)$ and $h_{k}\left(M_{j}\right)$ are, respectively, the (homotopy) index of $\left(S \times E_{k}\right) / G$ and $\left(M_{j} \times E_{k}\right) / G, P_{t}(\cdot)$ is the Poincare series which represents the Cech-cohomology of any element in the equivalence class $h_{k}(S)$ or $h_{k}\left(M_{j}\right)$ and $Q_{t}^{k}$ is a series with nonnegative integer coefficients.

We recall that $P_{t}\left(h_{k}(S)\right)$ (resp. $\left.P_{t}\left(h_{k}\left(M_{j}\right)\right)\right)$ can be computed from any index pair $\left(\left(N \times E_{k}\right) / G,\left(N^{-} \times E_{k}\right) / G\right)$ of $\left(S \times E_{k}\right) / G\left(\right.$ resp. $\left.\left(M_{j} \times E_{k}\right) / G\right)$ if $\left(N, N^{-}\right)$is a $G$-invariant index pair for $S$ (resp. $M_{j}$ ).

Now, if we take the limit in (2.2), $k \rightarrow \infty$, using the stabilization property of the cohomology for the classifying spaces of compact topological groups [11, Chapter III] $]^{3}$ we obtain

$$
\sum_{j=1}^{n} P_{t}^{G}\left(h\left(M_{j}\right)\right)=P_{t}^{G}(h(S))+(1+t) Q_{t}^{G} .
$$

In (2.3) $P_{t}^{G}(h(S))$ (resp. $P_{t}^{G}\left(h\left(M_{j}\right)\right)$ ) represents the cohomology of the pair $\left((N \times E) / G,\left(N^{-} \times E\right) / G\right)$, if $\left(N, N^{-}\right)$is a $G$-invariant index pair for $S$ (resp. $\left.M_{j}\right)$, that is the equivariant cohomology of the pair $\left(N, N^{-}\right){ }^{4}$

If $I$ is a $G$-invariant isolated invariant set and $\left(N, N^{-}\right)$is a $G$-invariant index pair for $I$, the homotopy type of the pair $\left((N \times E) / G,\left(N^{-} \times E\right) / G\right)$, will be denoted by $h_{G}(I)$ and called the equivariant-(homotopy) index of $I$.

Thus (2.3) could be written

$$
\sum_{j=1}^{n} P_{t}\left(h_{G}\left(M_{j}\right)\right)=P_{t}\left(h_{G}(S)\right)+(1+t) Q_{t}^{G} .
$$

Proposition 2.2. If I is a G-invariant isolated invariant set and the action of $G$ on $I$ is free then $P_{t}\left(h_{G}(I)\right)=P_{t}(h(I / G))$ where $h(I / G)$ is the (homotopy) index of $I / G$ in the quotient flow on $\Gamma / G$.

Proof. Since the action on $I$ is free and the set of the points in $\Gamma$ on which the action is free is open in $\Gamma$ it is possible to find an open neighborhood $V$ of $I$ in $\Gamma$ on which the action is also free.

Then we can take an index pair $\left(N, N^{-}\right)$with $N \subset V$, so that the action of $G$ is free on $N$. But, whenever the action on a space is free the equivariant cohomology coincides with the ordinary cohomology of the quotient space. So we have

$$
\begin{aligned}
P_{t}\left(h_{G}(I)\right) & =P_{t}\left((N \times E) / G,\left(N^{-} \times E\right) / G\right) \\
& =P_{t}\left(N / G, N^{-} / G\right)=P_{t}(h(I / G)) .
\end{aligned}
$$

We close this section by showing that if $G$ is a compact Lie group and we have a gradient flow on a $G$-invariant compact manifold $M$, induced by a nondegenerate $G$-invariant smooth function $f(2.3)$ generalizes the (classical) equivariant Morse inequalities described in [1 and 4].

\footnotetext{
${ }^{3}$ If $E=\lim _{k \rightarrow \infty} E_{k}$ and $B G=\lim _{k \rightarrow \infty} E_{k} / G$ then for each $i \in N$ there exists $m(i) \in N$, such that $k \geqslant m(i) \Rightarrow H^{i}(E) \cong H^{i}\left(E_{k}\right)$ and $H^{i}(B G) \cong H^{i}\left(E_{k} / G\right)$.

${ }^{4}$ If $G$ acts on a space $X$ and $E$ is the total space in the bundle (2.1) the equivariant cohomology of $X$, $H_{G}(X)$, is, by definition, the cohomology of $(X \times E) / G=X_{G}$.
} 
In fact, considering again the bundle (2.1) the function $f$ can be extended to a $G$-invariant function on $M \times E$ and hence projected to a function $f_{E}$ on $(M \times E) / G=M_{G}$.

The important thing is that in doing this we do not lose the nondegeneracy of $f$, that is $f_{E}$ is still nondegenerate on $M_{G}$. Moreover, if $T$ is a nondegenerate critical manifold ${ }^{5}$ of $f$ on $M,(T \times E) / G$ is a nondegenerate critical manifold of $f_{E}$ and the Morse indices of $T$ relative to $f$ and $(T \times E) / G$ relative to $f_{E}$ are equal [1].

Since a nondegenerate $G$-invariant function has only a finite number of critical orbits, which are nondegenerate critical manifolds, we can take as a Morse decomposition of $f$ the one given by these critical orbits, ordering them according to the values of $f[8,16]$.

From (2.3) we get

$$
\sum_{T} P_{t}^{G}(h(T))=P_{t}^{G}(M)+(1+t) Q_{t}^{G}
$$

where the summation is extended to all the critical orbits of $f$.

Because of the lack of compactness (2.5) is obtained using the same approximation method employed in deriving (2.3). That is $P_{t}^{G}(h(T))=\lim _{k \rightarrow \infty} P_{t}\left(h_{k}(T)\right)$.

But for nondegenerate critical manifolds the (homotopy) index can be computed more directly $[1,16]$ :

$$
P_{t}\left(h_{k}(T)\right)=t^{\lambda} T P_{t}\left(\left(T \times E_{k}\right) / G, \mathcal{O}^{-} \otimes K\right)
$$

where $\lambda_{T}$ is the number of negative eigenvalues of the hessian of $f$ in the direction normal to $\left(T \times E_{k}\right) / G$ (which is the same as for $T$ ), $K$ is a ring and $P_{t}\left(\cdot, \mathcal{O}^{-} \otimes K\right.$ ) denotes the cohomology with local coefficients. More precisely $\mathcal{O}^{-}$is the orientation bundle of $\nu^{-}(T)$, which is the part of the normal bundle $\nu(T)$ spanned by the negative eigenvalues of the hessian of $f$ on $\nu(T)$. In particular, if $\nu^{-}(T)$ is orientable $P_{t}\left(T, \mathcal{O}^{-} \otimes K\right)$ is the cohomology with ordinary coefficients.

Then passing to the limit in (2.6), for each critical orbit $T,(2.5)$ becomes

$$
\sum_{T} t^{\lambda_{T}} P_{t}^{G}\left(T, \mathcal{O}^{-} \otimes K\right)=P_{t}^{G}(M)+(1+t) Q_{t}^{G}
$$

where $P_{t}^{G}(M)$ (resp. $P_{t}^{G}(T)$ ) represents the equivariant cohomology of $M$ (resp. $T$ ).

Moreover each $T$, being a single orbit, is homeomorphic to $G / H$, where $H$ is the isotropy group 6 of each point of $T$. Then we have

$$
(T \times E) / G \cong(G / H \times E) / G \cong E / H \cong B H .
$$

The last relation holds because if $E$ is the total space for a universal $G$-bundle, $E$ is also the total space for a universal $H$-bundle; hence $E / H$ is homotopically equivalent to $B H$, the classifying space of $H$.

\footnotetext{
${ }^{5} T$ is a nondegenerate critical manifold of $f$ if [5]

(i) each point $p \in T$ is a critical point of $f$.

(ii) the hessian of $f$ is nondegenerate in the normal direction to $T$.

${ }^{6}$ The isotropy group of a point $x \in T$ is the subgroup of $G$ defined by $G_{x}=\{g \in G \mid g x=x\}$.
} 
Then (2.7) becomes

$$
\sum_{T} t^{\lambda_{T}} P_{t}\left(B H, \mathcal{O}^{-} \times K\right)=P_{t}^{G}(M)+(1+t) Q_{t}^{G}
$$

Moreover, if $H$ is connected, we do not need to use local coefficients because, in this case, $B H$ is simply connected and every bundle over a simply connected base is orientable.

3. An application to the $N$-body problem. Let us denote by $q=\left(q_{1}, \ldots, q_{N}\right) \in \mathbf{R}^{3 N}$ the positions of $N$ bodies with masses $m_{1}, \ldots, m_{N}$ respectively.

The potential energy is the function

$$
V(q)=-\sum_{i<j} \frac{m_{i} m_{j}}{\left|q_{i}-q_{j}\right|}
$$

Keeping the same notations as in [15] we denote by $\mathscr{E}$ the manifold

$$
\mathscr{E}=\left\{q \in \mathbf{R}^{3 N}: \sum_{i=1}^{N} m_{i} q_{i}=0 \text { and } \sum_{i=1}^{N} m_{i}\left|q_{i}\right|^{2}=1\right\}
$$

and by $\Delta$ the set $\left\{q \in \mathbf{R}^{3 N}\right.$ : there exists $i \neq j$ such that $\left.q_{i}=q_{j}\right\}$.

We are interested in studying the critical points of $V$ restricted to the manifold $M=\mathscr{E} \backslash \Delta$.

As shown in [15], this is equivalent to studying the central configurations of $N$ bodies.

We also consider the cases when the bodies lay on a fixed line or on a fixed plane. Let us examine both cases.

When the bodies are constrained to move on a fixed line the problem is called collinear. In this case the manifold $T$ corresponding to $\mathscr{E} \backslash \Delta$ is $N-2$ dimensional and is invariant, together with the potential $V$, under the diagonal action of the group $Z_{2}$, given by the reflection with respect to the origin.

If the bodies are constrained to move on a fixed plane the problem is called coplanar and the manifold $Y$ corresponding to $\mathscr{E} \backslash \Delta$ is $2 N-3$ dimensional. The problem is invariant under the action of the group $O(2)$, given by the rotations in that plane plus the reflections with respect to the lines through the origin.

In this case any collinear configuration has a nontrivial isotropy group isomorphic to $Z_{2}$, while the action is free on the planar configurations. If we restrict our attention only to the action of $S O(2)=S^{1}$ on $Y$, then the action is free also on the collinear configurations.

The coplanar problem has been studied by Palmore by applying Morse theory to the quotient space $Y / S^{1}[14]$.

If there is no restriction on the motion of the $N$ bodies the problem is called spatial.

The manifold $M$ is $3 N-4$ dimensional and is invariant for the action of $O(3)$, as well as the potential $V(q)$. 
The nontrivial isotropy groups are $O(2)$ and $Z_{2}$ for the collinear and planar configurations, respectively.

To consider only the action of $S O(3) \subset O(3)$ does not make the action free but reduces the isotropy groups to $S^{1}$ and the identity, respectively.

The spatial problem has been studied in [15] by applying the equivariant Morse theory to the $S O(3)$-equivariant flow $\dot{q}=\left.\nabla V\right|_{M}$.

When the masses $\left(m_{1}, \ldots, m_{N}\right)$ are such that the corresponding potential energy has only nondegenerate critical orbits the equivariant Morse relations obtained in [15] are

$$
\left\{\begin{aligned}
\sum_{\lambda=0}^{2 N-5}\left(\alpha_{\lambda}+\beta_{\lambda}\right) t^{\lambda}+\left(\frac{N !}{2}\right) \frac{t^{2 N-4}}{1-t^{2}} & =\frac{\left(1+t^{2}\right) \cdots\left(1+(N-1) t^{2}\right)}{1-t^{4}}+Q_{t}(V) \\
& =\frac{1}{1-t^{2}} \sum_{i=0}^{N-2} \gamma_{2 i} t^{2 i}+(1+t) Q_{t}(V)
\end{aligned}\right.
$$

where

(i) $\alpha_{\lambda}$ is the number of spatial critical orbits, whose points have index $\lambda,{ }^{7}$

(ii) $\beta_{\lambda}$ is the number of planar critical orbits whose points have index $\lambda$,

(iii) $(N ! / 2) t^{2 N-4} /\left(1-t^{2}\right)$ is the part related to the collinear configurations (according to (2.8)),

(iv) $\sum_{i=0}^{N-2} \gamma_{2 i}=N ! / 2$.

From (3.1) the following estimate holds [15]:

$$
\alpha_{2 j}+\beta_{2 j} \geqslant \gamma_{0}+\gamma_{2}+\cdots+\gamma_{2 j}, \quad 0 \leqslant 2 j \leqslant 2 N-5 .
$$

As a consequence of Proposition 2.2 we have

Proposition 3.1. Equivariant Morse relations of (3.1) type hold also when the potential energy has a finite number of degenerate critical orbits.

Proof. Since the action of $S O(3)$ is free on the spatial and planar configurations the equivariant index of each critical orbit coincides with the index in the quotient space $M / S O(3)$.

Then this index is expressed by a polynomial of the type $P(t)=\sum_{i=0}^{2 N-5} a_{i} t^{i}$; so that summing up all the polynomials we obtain the expression (3.1) where, of course, $\lambda$ does not represent the number of positive eigenvalues of the hessian of $V$ any more.

The previous proposition implies that if each $P(t)$ is a monomial $\alpha_{\lambda} t^{\lambda}$ (or $\beta_{\lambda} t^{\lambda}$ ), from (3.2) we deduce an estimate of the number of spatial and planar central configurations, even in the presence of degenerate critical orbits.

Now let us compare (3.1) with the Morse inequalities for the planar problem [14].

\footnotetext{
${ }^{7}$ Here the word index means the number of positive eigenvalues of the hessian of $V$ at each point of a critical orbit.
} 
Applying Morse theory in the quotient manifold $Y / S^{1}$ one gets

$$
\left\{\begin{aligned}
& \sum_{i=0}^{N-2} b_{i} t^{i}+\left(\frac{N !}{2}\right) t^{N-2}=(1+2 t)(1+3 t) \cdots(1+(N-1) t)+(1+t) Q_{t}(V) \\
&=\sum_{i=0}^{N-2} \delta_{i} t^{i}+(1+t) Q_{t}(V), \\
& \sum_{i=0}^{N-2}=\frac{N !}{2} .
\end{aligned}\right.
$$

From (3.3), equating the coefficients of the same powers and denoting by $2 p$ the number of $S^{1}$-critical orbits of planar configurations (except the collinear ones) we get

$$
2 p \geqslant 1+\delta_{1}+\cdots+\delta_{N-3}+\left(N ! / 2-\delta_{N-2}\right) .
$$

Since $N ! / 2=1+\delta_{1}+\cdots+\delta_{N-2}$, we have

$$
2 p \geqslant 2\left(1+\delta_{1}+\cdots+\delta_{N-3}\right) .
$$

But, as we observed before, the coplanar problem is invariant under the $O(2)$ symmetry and $O(2)$ is contained in $S O(3)$. Then the number of $S O(3)$-critical orbits coming from planar configurations is

$$
p \geqslant 1+\delta_{1}+\cdots+\delta_{N-3}=m .
$$

From (3.2), denoting by $s$ the number of $S O(3)$-critical orbits of spatial configurations (i.e. except the collinear and coplanar ones) we get

$$
s+p \geqslant 1+\left(1+\gamma_{2}\right)+\cdots+\left(1+\gamma_{2}+\cdots+\gamma_{2 N-6}\right)=\mu .
$$

Since $\delta_{i}=\gamma_{2 i}, 0 \leqslant i \leqslant N-3$, we have

$$
\mu-m \geqslant 1+\left(1+\delta_{1}\right)+\cdots+\left(1+\delta_{1}+\cdots+\delta_{N-4}\right)
$$

so that $\mu-m \rightarrow \infty$ for $N \rightarrow \infty$.

The estimate (3.7) is very important because it shows that from the equivariant Morse inequalities one deduces the presence of many more central configurations than one could guess from the Morse inequalities for the planar problem.

This is obvious if one believes that there are "many" spatial central configurations that are not coplanar, which is still an open problem.

As pointed out in [15] a better estimate of the index of the coplanar configurations, together with (3.2) and (3.7) could solve this problem, allowing a distinction between the coplanar and spatial configurations, in the same way as it happens for the collinear configurations.

We end by showing that the collinear problem is a "repeller" in the gradient flow $\dot{q}=\left.\nabla V\right|_{M}$.

First of all we observe that even if the manifold $M=\mathscr{E} \backslash \Delta$ is not compact, the critical points of $\left.V\right|_{M}$ lie in a compact contained in $M$. Actually, it is possible to prove that there exists a neighborhood of $\Delta$ in $\mathscr{E}$ in which there are no critical points of $\left.V\right|_{M}[18]$.

Hence the set $S=\{q \in M \mid \operatorname{cl}(q \cdot \mathbf{R})$ is compact in $M\}$ is compact. 
We set $S_{0}=\{q \in S \mid q$ is a collinear configuration $\}$. $S_{0}$ is a compact invariant set relative to $S$ and we can prove the following theorem.

THEOREM 3.1. $S_{0}$ is a repeller relative to $S .^{8}$

Proof. By a collinear configuration we mean a configuration where all the masses are positioned on a line through the origin in $\mathbf{R}^{3}$.

In [15] it has been proved that if $\bar{q}$ is a collinear central configuration the hessian $H V(\bar{q})$ is positive definite in each direction normal to $S_{0} \subset S$.

The number of these normal directions is $2 N-4$. This implies that the (homotopy) index $h(\bar{q})$ relative to $S$ is equal to

$$
h(\bar{q}, S)=h\left(\bar{q}, S_{0}\right) \wedge \Sigma^{2 N-4,},
$$

where $h\left(\bar{q}, S_{0}\right)$ is the (homotopy) index of $\bar{q}$ relative to $S_{0}$.

Moreover if $q$ is any point in $S$, its $\omega$-limit, $\omega(q)$ must be a critical point of $\left.V\right|_{M}$, since the flow is a gradient flow.

Thus from (3.8) we have that if $q \in S$ and $\omega(q) \in S_{0}$, then $q \in S_{0}$, because the critical points of $\left.V\right|_{M}$ in $S_{0}$ are "repellers" in the direction normal to $S_{0}$.

We prove that $S_{0}$ is a repeller, relative to $S$, by proving that there exists a compact neighborhood $N$ of $S_{0}$, relative to $S$, such that $I(N)$, the maximal invariant set in $N$, is $S_{0}$ and each boundary point of $N$ leaves $N$ in forward time.

As shown in [7] this implies that $S_{0}$ is a repeller relative to $S$.

Let $N$ be a compact neighborhood of $S_{0}$ in $S$, disjoint from all the remaining critical points.

Thus the maximal invariant set in $N$ is $S_{0}$. In fact if $q \in N$ and $q \cdot \mathbf{R} \in N$ then $\omega(q) \in N$ and must be a critical point of $\left.V\right|_{M}$ in $S_{0}$. From (3.8) $q$ belongs to $S_{0}$.

If $q \in \partial N$, then $q \notin S_{0}$ and hence $\omega(q) \notin S_{0}$. Then there exists $t>0$ such that $q \cdot t \notin N$, otherwise $\omega(q)$ would belong to $N$ and would be a critical point of $\left.V\right|_{M}$ in $S_{0}$. So each boundary point of $N$ eventually leaves $N$ in forward time. The proof is complete.

\section{REFERENCES}

1. M. F. Atiyah and R. Bott, The Yang-Mills equations over Riemann surfaces, Philos. Trans. Roy. Soc. London A 308 (1982), 523-615.

2. V. Benci, A geometrical index for the group $S^{1}$ and some applications to the study of periodic solutions of ordinary differential equations, Comm. Pure Appl. Math. 34 (1981), 393-432.

3. V. Benci and F. Pacella, Morse theory for symmetric functionals on the sphere and an application to a bifurcation problem, Nonlinear Analysis: Theory, Methods, and Applications 9 (1985), 763-773.

4. R. Bott, Lectures on Morse theory, old and new, Bull. Amer. Math. Soc. 7 (1982), 331-358.

5. __ Nondegenerate critical manifolds, Ann. of Math. (2) 60 (1954), 248-261.

6. G. E. Bredon, Introduction to compact transformation groups, Academic Press, New York, 1972.

7. C. C. Conley, Isolated invariant sets and the Morse index, CBMS Regional Conf. Ser. in Math., no. 38, Amer. Math. Soc., Providence, R. I., 1978.

\footnotetext{
${ }^{8}$ To say that $S_{0}$ is a repeller relative to $S$ means that there exists a neighborhood $U$ of $S_{0}$ in $S$ such that $\omega(U)=S_{0}[7]$.

${ }^{9} \wedge$ denotes the (smash) product of two pointed spaces and $\Sigma^{2 N-4}$ denotes the pointed $(2 N-4)$-sphere (for further details see [7]).
} 
8. C. C. Conley and E. Zehnder, Morse type index theory for flows and periodic solutions for hamiltonian equations, Comm. Pure Appl. Math. 37 (1984), 207-253.

9. E. R. Fadell and S. Husseini, Relative cohomological index theories (to appear).

10. E. R. Fadell and P. H. Rabinowitz, Generalized cohomological index theories for Lie group actions with an application to bifurcation questions for hamiltonian systems, Invent. Math. 45 (1978), 139-174.

11. K. H. Hofmann and P. S. Mostert, Cohomology theories for compact abelian groups, Springer-Verlag, New York, 1973.

12. D. Husemoller, Fibre bundles, Springer-Verlag, New York, 1966.

13. J. Milnor, Morse theory, Ann. of Math. Studies, no. 51, Princeton Univ. Press, Princeton, N.J., 1963.

14. J. I. Palmore, Classifying relative equilibria, Bull. Amer. Math. Soc. 81 (1975), 489-491.

15. F. Pacella, Central configurations of the N-body problem via the equivariant Morse theory, Arch. Rational Mech. Anal. (to appear).

16. , Morse theory for flows in the presence of a symmetry group, M. R. C. Rep. No. 2717, July 1984.

17. J. Smoller, Shock waves and reaction-diffusion equations, Springer-Verlag, Berlin and New York, 1983.

18. M. Shub, Appendix to Smale's paper: Diagonals and relative equilibria (Proc. Nuffic Summer School), Lecture Notes in Math., vol. 197, Springer, Berlin and New York, 1971, pp. 199-201.

19. E. H. Spanier, Algebraic topology, McGraw-Hill, New York, 1966.

20. A. G. Wasserman, Equivariant differential topology, Topology 8 (1969), 127-150.

Dipartimento di Matematica e Applicazioni, Via Mezzocannone 8, 80134 Napoli, Italy 